\title{
Tunable disorder and localization in the rare-earth nickelates
}

\author{
Changan Wang, $, 1,2,3, *$ Ching-Hao Chang, ${ }^{4}{ }^{\dagger}$ Angus Huang, ${ }^{5}$ Pei-Chun \\ Wang, ${ }^{6}$ Ping-Chun Wu, ${ }^{6}$ Lin Yang, ${ }^{7}$ Chi Xu, ${ }^{1,2}$ Parul Pandey, ${ }^{1}$ Min \\ Zeng, ${ }^{7}$ Roman Böttger, ${ }^{1}$ Horng-Tay Jeng, ${ }^{5,8}$ Yu-Jia Zeng, ${ }^{3}$ Manfred \\ Helm, ${ }^{1,2}$ Ying-Hao Chu, ${ }^{6,9}$ R. Ganesh, ${ }^{10}$ and Shengqiang Zhou ${ }^{1}$ \\ ${ }^{1}$ Helmholtz-Zentrum-Dresden-Rossendorf, \\ Bautzner Landstraße 400, 01328 Dresden, Germany \\ ${ }^{2}$ Technische Universität Dresden, 01062 Dresden, Germany \\ ${ }^{3}$ Shenzhen Key Laboratory of Laser Engineering, \\ College of Optoelectronic Engineering, \\ Shenzhen University, 518060 Shenzhen, China \\ ${ }^{4}$ Leibniz-Institute for Solid State and Materials Research, \\ Helmholtzstraße 20, 01069 Dresden, Germany \\ ${ }^{5}$ Department of Physics, National Tsing Hua University, Hsinchu 30043, Taiwan \\ ${ }^{6}$ Department of Materials Science and Engineering, \\ National Chiao Tung University, Hsinchu, Taiwan \\ ${ }^{7}$ Institute for Advanced Materials and Guangdong Provincial Key \\ Laboratory of Quantum Engineering and Quantum Materials, \\ South China Normal University, Guangzhou 51006, China \\ ${ }^{8}$ Institute of Physics, Academia Sinica, Taipei 11529, Taiwan \\ ${ }^{9}$ Center for Emergent Functional Matter Science, \\ National Chiao Tung University, Hsinchu 30010, Taiwan \\ ${ }^{10}$ The Institute of Mathematical Sciences, \\ HBNI, C I T Campus, Chennai 600113, India
}

(Dated: April 14, 2021) 


\begin{abstract}
The rare-earth nickelates are a rich playground for transport properties, known to host nonFermi liquid character, resistance saturation and metal-insulator transitions. We report a study of transport in $\mathrm{LaNiO}_{3}$ in the presence of tunable disorder induced by irradiation. While pristine $\mathrm{LaNiO}_{3}$ samples are metallic, highly irradiated samples show insulating behaviour at all temperatures. Using irradiation fluence as a tuning handle, we uncover an intermediate region hosting a metal-insulator transition. This transition falls within the Mott-Ioffe-Regel regime wherein the mean free path is comparable to lattice spacing. In the high temperature metallic regime, we find a transition from non-Fermi liquid to a Fermi-liquid-like character. On the insulating side of the metal-insulator transition, we find behaviour that is consistent with weak localization. This is reflected in magnetoresistance that scales with the square of the field and in resistivity. In the highly irradiated insulating samples, we find good agreement with variable range hopping, consistent with Anderson localization. We find qualitatively similar behaviour in thick $\mathrm{PrNiO}_{3}$ films as well. Our results demonstrate that ion irradiation can be used to tailor transport, serving as an excellent tool to study the physics of localization.
\end{abstract}


Introduction A central question in condensed matter physics is the distinction between insulators and metals. In this context, Anderson localization [1-3] is a key paradigm that explains how a disordered potential can suppress transport via quantum interference. This is particularly interesting in three spatial dimensions wherein a threshold disorder strength is required to localize electrons. Direct simulations of 3D Anderson localization have been achieved in areas as diverse as light [4], sound [5] and ultracold atom gases [6, 7]. Signatures of localization have also been seen in solid state systems, mainly in doped semiconductors [8-11]. In this letter, we demonstrate that irradiation can serve as a tuning knob to study a wide range of localization phenomena.

We study transport in the rare-earth nickelates [12]. This family of materials hosts several complex phenomena such as non-Fermi liquid character [13], charge fluctuations [14], magnetic correlations [15] and a width-tuned MIT (metal-insulator transition) [16]. As the underlying physics continues to be debated, we show that these materials can be tuned across a wide range of localization phenomena by irradiation. We explain our results using several known paradigms: the Mott-Ioffe-Regel limit, weak localization and variable range hopping.

Since the formulation of the iconic Drude model, it is known that a key quantity that determines conductivity is mean-free path - the average distance travelled by electrons between successive collisions. The collisions themselves can be elastic (between electrons and crystal defects) or inelastic (electron-electron or electron-phonon scattering). We use ion irradiation to induce defects $[17,18]$, thereby directly controlling the elastic scattering rate. Our results demonstrate that this provides a superior tuning knob for transport properties when compared to tuning strain, doping or thickness. Unlike these methods, irradiation tunes the mean free path without significantly affecting other system parameters such as dimensionality and carrier concentration.

Experimental details $\mathrm{LaNiO}_{3}$ (LNO) films of width $50 \mathrm{~nm}$ were epitaxially grown on $\mathrm{SrTiO}_{3}(001)$ single crystals $\left(a_{\text {sub }}=3.905 \AA\right.$ ) by pulsed laser deposition. The films were then irradiated by $6 \mathrm{keV}$ He ions with four different fluences, $\Phi=1,1.75,2.5$, to $5 \times 10^{15} \mathrm{He} / \mathrm{cm}^{2}$. Our work is, in part, motivated by early studies on ternary borides where He-irradiation was shown to affect transport [19]. The He ions create point defects with a density that increases with fluence. Helium's nobility ensures that no extra holes or electrons are doped into the films. It does induce strain that is relieved by an increase of out-of-plane lattice constant 
(see Supplementary Figure 1), but this doesn't significantly affect carrier density. The pristine and irradiated samples were characterized by x-ray diffraction (XRD) (PANalytical XPert PRO diffractometer) using $\mathrm{Cu} \mathrm{K} \alpha$ radiation. Transport properties were measured using a Van-der-Paul geometry and a constant current source. To measure temperature dependence, temperature was swept at a slow rate of 1-2 K/min with a Lakeshore 332 temperature controller. We have performed the same measurements on $\mathrm{PrNiO}_{3}$ as well, with qualitatively similar results.

Resistivity Metallic and insulating behaviours are distinguished by the temperature dependence of resistivity. Fig. 1(a) shows resistivity vs. temperature for the pristine film as well as irradiated samples. The pristine film as well that with the lowest fluence are both metallic at all temperatures. In contrast, the sample with the largest fluence $\left(\Phi=5 \times 10^{15}\right.$ $\mathrm{He} / \mathrm{cm}^{2}$ ) exhibits insulating behavior at all temperatures. At intermediate fluences, we see a clear minimum in the resistivity, indicating an MIT. Here, we use the term MIT to denote a thermal crossover where the the slope of resistivity vs. temperature changes sign. We observe that the MIT temperature increases with increasing fluence.

Metallic side of the MIT In a typical metal, resistivity increases with temperature according to

$$
\rho_{\text {ideal }}(T)=\rho_{0}+A T^{n}
$$

The resistivity at zero temperature, $\rho_{0}$, arises due to elastic scattering from defects in the crystal - a measure of disorder strength. In contrast, the temperature dependent contribution arises largely from inelastic electron-electron and electron-phonon scattering [20]. In a Fermi liquid, when electron-electron scattering is dominant, the exponent is $n=2$ leading to a characteristic quadratic behaviour. The interplay of elastic and inelastic scattering can be described in terms of the mean free path, $\ell$, with $\rho \propto \ell^{-1}[21]$,

$$
\frac{1}{\ell}=\frac{1}{\ell_{e}}+\frac{1}{\ell_{i n}}
$$

as the elastic $\left(\ell_{e}\right)$ and inelastic $\left(\ell_{i n}\right)$ scattering rates combine additively. With increasing temperature, $\ell_{i n}$ decreases leading to increasing resistivity. This increase cannot go on indefinitely as the mean free path cannot be shorter than the lattice spacing. The regime when the mean free path is comparable to the lattice spacing is called the Mott-Ioffe-Regel limit $[22,23]$. This leads to an upper bound on the resistivity, $\rho_{\text {sat }}$, which can be thought of arising from Heisenberg's uncertainty principle [22, 24, 25]. The overall resistivity is given 

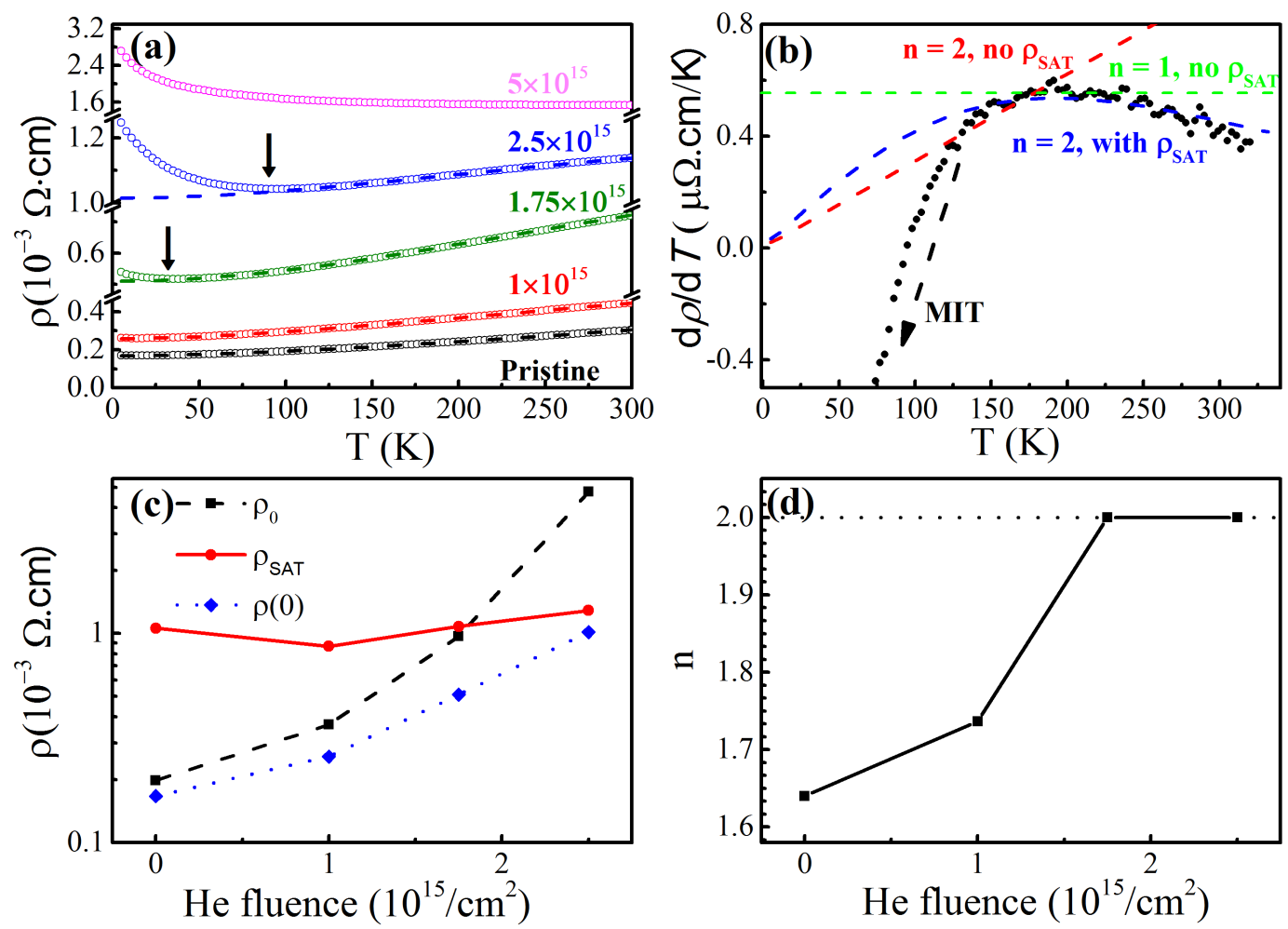

FIG. 1: (a) Resistivity vs. temperatures of LNO samples with the increasing fluence of irradiation. Arrows mark MITs. The dashed lines are fits of data in the metallic region using Eq. (1) and Eq. (3). (b) Temperature derivative of the resistivity as a function of temperature for the sample with a fluence $\Phi=2.5 \times 10^{15} \mathrm{He} / \mathrm{cm}^{2}$. The downturn at lower temperature represents the MIT. Red and green dashed lines are fits with $\rho_{\text {sat }}=0$, which do not describe the data well. (c) $\rho_{0}, \rho_{\text {sat }}$, and $\rho(T=0)$ obtained at different fluences. (d) Extracted exponents $n$ for different fluences.

by

$$
\rho^{-1}(T)=\rho_{\text {ideal }}^{-1}(T)+\rho_{\text {sat }}^{-1}
$$

This parallel-resistor formula is well known to describe a wide variety of metals [22].

Here, we find that nickelate films on the metallic side of the MIT are well described by a combination of Eqs. 3 and 1. We fit the resistivity data taking $\rho_{0}, \rho_{\text {sat }}, A$ and $n$ as fitting parameters. The obtained results are shown in Fig. 1(c,d). In order to obtain a reasonable fit, it is essential to include a saturation resistivity $\rho_{\text {sat }}$ using the parallel resistor formula of Eq. 3 [26]. This is brought out in Fig. 1(b) which shows the derivative of resistivity and the corresponding fitting curves. As shown in Fig. 1(c), the resistivity extrapolated to zero temperature, $\rho(0)$, exhibits the typical result of parallel resistor addition. For low fluences, 
when $\rho_{0} \ll \rho_{\text {sat }}, \rho(0)$ tracks $\rho_{0}$. At high fluences, when $\rho_{0} \gg \rho_{\text {sat }}, \rho(0)$ approaches $\rho_{\text {sat }}$.

As shown in Fig. 1(c), $\rho_{0}$ increases with fluence - consistent with our expectation that $\rho_{0}$ is determined by the density of defects which controls the elastic mean free path. As $\rho_{0}$ also depends on carrier density, we measured Hall resistivity on the pristine, $\Phi=1 \times 10^{15}$ and $\Phi=$ $1.75 \times 10^{15} \mathrm{He} / \mathrm{cm}^{2}$ samples at $75 \mathrm{~K}$ (all three samples are metallic at this temperature). The densities inferred from the Drude formula are 2.02, 1.40 and $1.31\left(\times 10^{22} \mathrm{~cm}^{-3}\right)$ respectively. This shows that changes in transport are driven by changing defect density, rather than carrier density. For example, the $\Phi=1 \times 10^{15}$ (always metallic) and $\Phi=1.75 \times 10^{15}$ (hosting an MIT) samples only differ by $\sim 7 \%$ in carrier density, but by $\sim 60 \%$ in $\rho_{0}$.

The saturation resistivity $\rho_{\text {sat }}$ is more or less independent of fluence, in line with our expectation that $\rho_{\text {sat }}$ is an intrinsic material property that depends on the lattice constant (and perhaps the carrier density). Earlier studies have suggested that $\rho_{\text {sat }}$ in nickelate films is sensitive to the in-plane strain [26] as it modifies bands near the Fermi energy [27]. In our samples, the in-plane strain is fixed by the STO substrate and does not vary with irradiation (see Supplementary Figure 2). Indeed, our $\rho_{\text {sat }}$ value is close to that seen in thick RNO films $[26]$.

The fluence-dependence of $\rho_{0}$ and $\rho_{\text {sat }}$ indicates progression towards the Mott-Ioffe-Regel limit and beyond. When $\rho_{0}$ exceeds $\rho_{\text {sat }}$ at a fluence of $\Phi \approx 1.75 \times 10^{15} \mathrm{He} / \mathrm{cm}^{2}$, it suggests that the elastic mean free path becomes comparable to the lattice spacing (we support this assertion with further arguments below). Surprisingly, this is reflected in the exponent $n$, plotted in Fig. 1(d). For low fluences, the exponent is $n \approx 1.6$ indicating non-Fermi liquid (NFL) behaviour, known to be present in LNO. This could possible arise from lattice [13], charge [14] or magnetic fluctuations [15, 28]. At large fluences with $\Phi \gtrsim 1.75 \times 10^{15}$ $\mathrm{He} / \mathrm{cm}^{2}$, we find $n \approx 2$ suggesting Fermi liquid (FL) character. Remarkably, this NFL-FL transition coincides with the Mott-Ioffe-Regel (MIR) limit as suggested by $\rho_{0}$ and $\rho_{\text {sat }}$ values. This suggests that a large disorder concentration is required to suppress magnetic/lattice fluctuations. NFL character in the nickelates and in heterostructures has seen a surge of interest with many recent experimental studies [26, 29, 30]. Our results suggest that this behaviour can be tuned by disorder concentration and indeed, vanishes in the MIR limit.

Insulating side of the MIT Having discussed the MIT seen at intermediate fluence values $\left(\Phi=1.75\right.$ and $\left.2.5 \times 10^{15} \mathrm{He} / \mathrm{cm}^{2}\right)$, we characterize the insulating side of the transition. In three dimensions, weak disorder (with strength below the threshold required for Anderson 
localization) leads to increased resistance due to quantum interference. In this regime, we argue that resistivity can be described by (see Supplementary Section 2)

$$
\rho(T)=\rho(0)-\alpha T^{n / 2}+\beta T^{n}-\gamma T^{3 n / 2},
$$

where $\alpha, \beta$, and $\gamma$ are coefficients related to the ratio of inelastic and elastic mean free paths, $\ell_{\text {in }} / \ell_{\mathrm{e}}$

$$
\frac{\ell_{\text {in }}(T)}{\ell_{\mathrm{e}}}=\frac{1}{\pi}\left(\frac{\beta^{2}}{\alpha^{2}}-\frac{\gamma}{\alpha}\right)^{-1} T^{-n} .
$$

In Fig. 2(a), the resistivity is shown with the best fit to Eq. (4). The best fit coefficient here is $n=1.5$, indicating that the inelastic scattering process is dominated by electron-electron interactions $\left(\ell_{\text {in }} \propto T^{-2}\right)[20,31]$ rather than electron-phonon interactions $\left(\ell_{\text {in }} \propto T^{-3}\right)$ [32]. Our result is consistent with previous observations in LNO films $[16,33]$. The ratio $\ell_{\text {in }} / \ell_{\mathrm{e}}$ from Eq. (5) obtained from best fit parameters is shown in Fig. 2(b). The increase of He fluence from $\Phi=1.75$ to $2.5 \times 10^{15} \mathrm{He} / \mathrm{cm}^{2}$ increases defect concentration, thereby decreasing $\ell_{e}$ and increasing $\ell_{i n} / \ell_{e}$. The obtained ratio $\ell_{\text {in }} / \ell_{\mathrm{e}}$ allows us to make quantitative statements about approaching the MIR limit. For instance, in the sample with $\Phi=2.5 \times 10^{15}$ $\mathrm{He} / \mathrm{cm}^{2}$ with temperature around $\sim 3 K$, we find $\ell_{\text {in }} / \ell_{\mathrm{e}} \gtrsim 100$ as shown in Fig. 2(b). In this temperature range, the inelastic mean free path $\left(\ell_{\text {in }}\right)$ for an LNO film is known to be approximately $40 \mathrm{~nm}[16,34]$. Therefore, the elastic mean free path $\left(\ell_{\mathrm{e}}\right)$ is as short as $4 \AA$, close to the LNO lattice constant $3.83 \AA$. This confirms that the film is indeed at the MIR limit. From the large value of $\ell_{\text {in }} / \ell_{\mathrm{e}}$, we also deduce that inelastic scattering processes are not important at low temperatures. It follows that the resistivity increase (with decreasing temperature) in this regime stems from elastic scattering and the associated quantum interference effects. This is consistent with our analysis assuming weak localization.

To further characterize the insulating side of the MIT, we present magnetotransport data. Fig. 3(a) shows magnetoresistance (MR) at different temperatures for the sample irradiated with fluence $\Phi=2.5 \times 10^{15} \mathrm{He} / \mathrm{cm}^{2}$. From the above discussion, this sample is expected to be close to the MIR limit. Resistance decreases with the application of a field as time-reversalbreaking disrupts quantum interference effects that are responsible for weak localization. In our samples that lie in the MIR regime, the elastic mean free path is a few Angstroms. The magnetic length, however, is $\ell_{B} \approx 26 / \sqrt{B(\text { tesla })} \mathrm{nm}$ which is always much greater than $\ell_{e}$. 


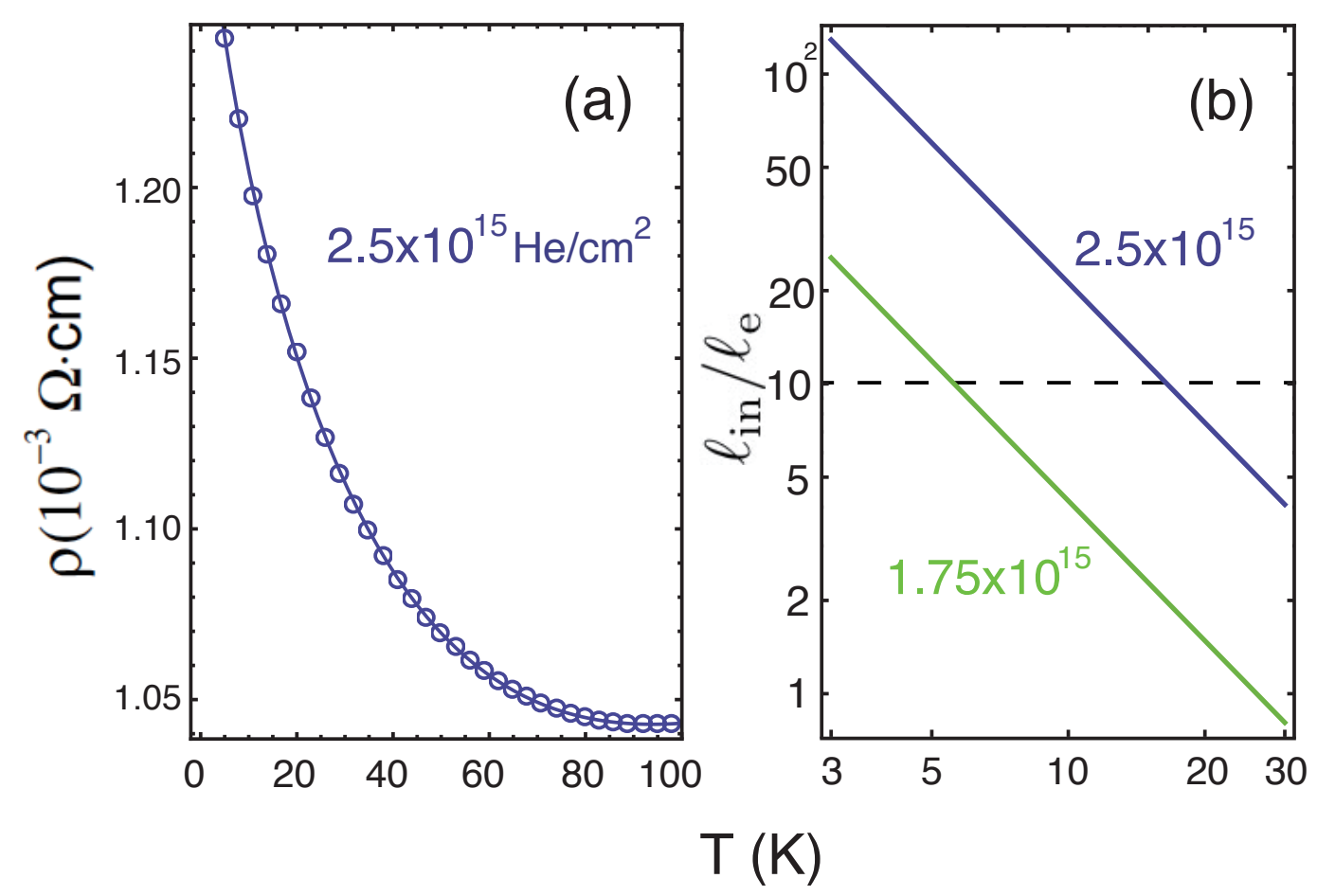

FIG. 2: (a) Increase of resistivity on the insulating side of the MIT, with a fit to Eq. 4. (2) A log-log plot of the ratio of mean free paths, obtained from the fitting parameters using Eq. 5 .

In this regime, with $\ell_{B} \gg \sqrt{\ell_{e} \times \ell_{i n}}$, the magnetoconductivity scales as [35]

$$
\Delta \sigma(\%)=\frac{\sigma(B, T)}{\sigma(0, T)}-1 \approx\left(\frac{\ell_{\mathrm{in}}}{\ell_{\mathrm{e}}}\right)^{3 / 2}\left(\frac{e \tau_{\mathrm{e}}}{m^{\star}}\right)^{2} B^{2},
$$

where $m^{\star}$ is the electron effective mass and $\tau_{\mathrm{e}}=\ell_{\mathrm{e}} / v_{F}$ is the elastic relaxation time proportional to the Fermi velocity $v_{F}$. Notably, the magnetoconductivity scales as the square of the field. Our data is in good agreement with this form as shown in Fig. 3(b); we see that $B^{2}$ scaling persists over a large field range, up to $5 T$. Moreover, the fitting parameter $A$ in Fig. 3(b) decreases with the increase of temperature. This is consistent with Eq. 6 as the ratio $\ell_{i n} / \ell_{e}$ decreases with temperature (see Fig. 2(b)). The magnetoconductivity of the sample irradiated with flux $\Phi=1.75 \times 10^{15} \mathrm{He} / \mathrm{cm}^{2}$ also shows $B^{2}$ scaling, as shown in the Supplementary Figure 3. We reiterate that the $B^{2}$ scaling is a consequence of the MIR limit. In pristine LNO, with a field of several Teslas, the magnetoconductivity scales as $B^{1 / 2}$ as the mean free path is longer than the magnetic length [34].

Insulating samples Having discussed both sides of the MIT seen at intermediate fluence, we discuss the sample with highest fluence, $\Phi=5 \times 10^{15} \mathrm{He} / \mathrm{cm}^{2}$ (see pink circles in Fig. 1(a)). 

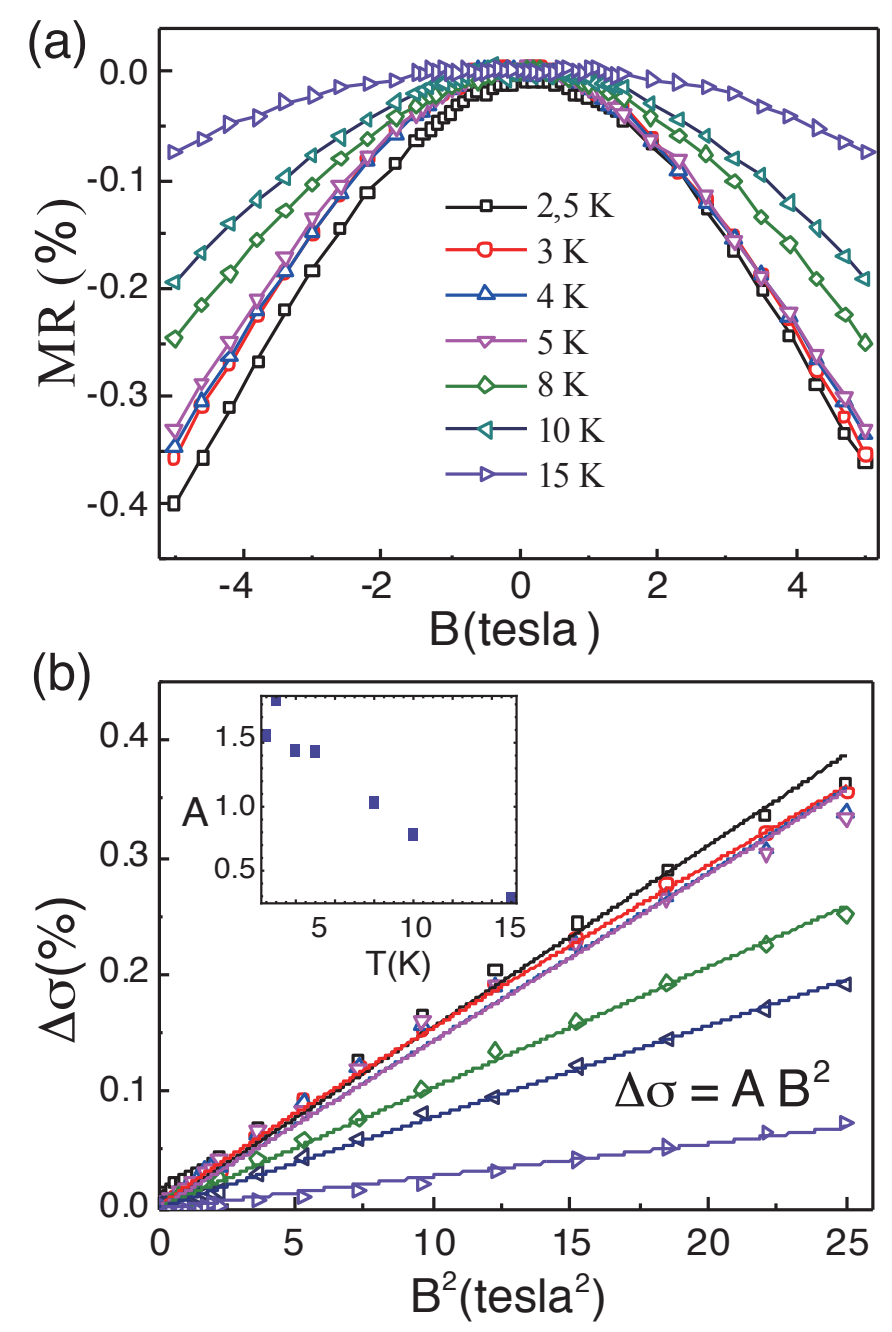

FIG. 3: (a) Magnetoresistance $(\{R(H)-R(0)\} / R(0))$ vs. field in the LNO sample irradiated with fluence $2.5 \times 10^{15} \mathrm{He} / \mathrm{cm}^{2}$ at different temperatures. (b) Fractional increase in magnetoconductivity $(\Delta \sigma(\%)=\{\sigma(B)-\sigma(0)\} / \sigma(0))$ vs. the square of the magnetic field. The inset shows the fitting parameter $A$ (unit: $10^{-4} /$ tesla $^{2}$ ) as a function of temperature.

This shows insulating behaviour at all temperatures. With high disorder concentration, we expect electrons to be Anderson-localized. Transport can only occur via hopping between localized states that are well-separated in position and energy, equivalent to a percolation process [36]. In this scenario, resistivity follows the variable range hopping (VRH) paradigm [37], with

$$
\rho(T)=C \exp \left(T_{0} / T\right)^{1 /(1+d)},
$$




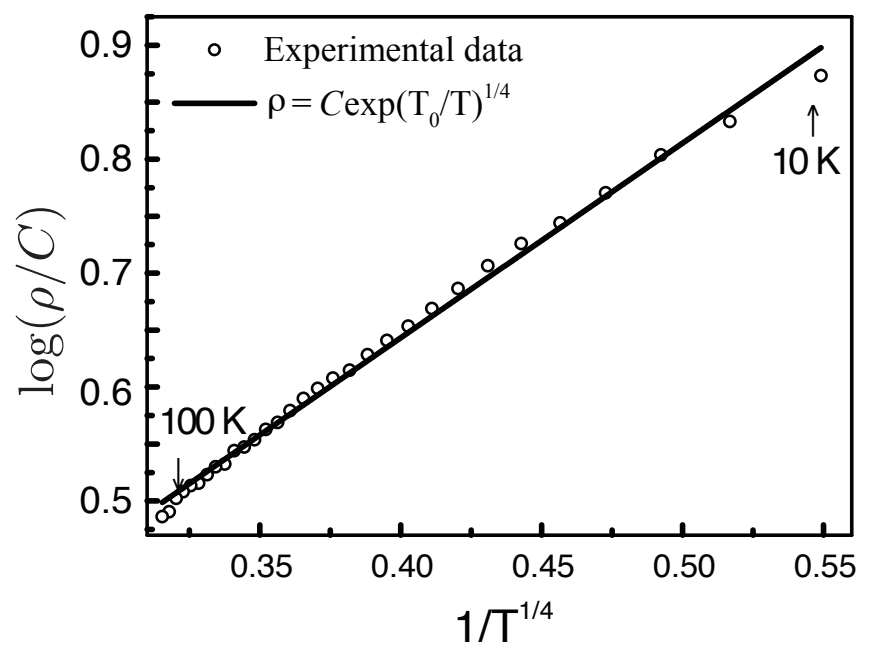

FIG. 4: Logarithm of resistivity vs. $\mathrm{T}^{-1 / 4}$ for the sample irradiated with fluence $5 \times 10^{15} \mathrm{He} / \mathrm{cm}^{2}$. The black line is a fit to the data between 10 and $100 \mathrm{~K}$.

where $d$ is the number of spatial dimensions. $T_{0}$ is a constant that depends on both the density of localized states and the spatial decay profiles of wavefunctions. Fig. 4 shows our resistivity data with a fit to Eq. 7 with $d=3$. We obtain a good fit from 10 to $100 \mathrm{~K}$. Earlier studies on insulating ultrathin LNO films that are insulating have shown good agreement with VRH behaviour, described by Eq. (7) with $d=2[16,38]$. The two-dimensionality arises from lateral confinement $[39,40]$. Here, as our film thickness is $50 \mathrm{~nm}$, much greater than the mean free path, we find three-dimensional character.

Discussion We have studied transport in nickelate films, with tunable disorder. With irradiation as a tuning knob, we see (i) a metallic phase in the clean regime, (ii) metal-insulator transitions for intermediate disorder, and (iii) insulating behaviour for high disorder. Our results on transport in $\mathrm{LaNiO}_{3}$ are robust, with the same qualitative results occurring in irradiated PrNiO3 as well (see Supplementary Figure 4), and can be compared with earlier studies on the nickelates using various tuning parameters: electric fields [41], oxygen vacancies [13], strain [26] and film thickness [16, 42]. We argue that irradiation provides a superior tuning knob as it changes only the elastic mean free path, without strongly affecting carrier density, dimensionality or band energies.

Our findings suggest that irradiated nickelates open a new window to the study of Anderson localization, as they allow for a clean handle on the mean free path. In particular, the following exciting question arises: can we detect a 'mobility edge' [43, 44] in a solid state 
system? In our samples with intermediate disorder, a new temperature scale (associated with the MIT) emerges. Measurements such as optical conductivity could reveal whether this is correlated with a mobility edge in the electronic spectrum. Such measurements are of great interest in the context of recent ultracold atom experiments $[45,46]$.

Acknowlegements C.W. thanks financial support from the China Scholarship Council (File No. 201606750007). C.-H.C. and P. P. acknowledge financial support from the German Research Foundation (Deutsche Forschungsgemeinschaft, Grant CH 2051/1-1 and ZH 225/6-1.). C.-H.C., A.H., and H.-T.J. acknowledge support from the National Center for Theoretical Sciences. R.G. thanks IFW Dresden for hospitality.

C.W. and C.-H.C. contributed equally to this work.

Author contributions C.W., C.-H.C. and S.Z. conceived the project. M. H. supervised the work. C.W. carried out the transport experiments. P.-C.W., P.-C.W., and Y.-H.C. prepared the samples. L.Y., M.Z. and Y.Z. performed XRD measurements. R.B. performed ion irradiation. C.W. and C.-H.C. carried out data analysis. C.-H.C. and R.G. developed the theoretical models, while A.H. and H.-T.J. performed band structure calculations. C.H.C., R.G. and S.Z. wrote the paper. All authors discussed the results and commented on the manuscript.

Competing Interests The authors declare no competing interests.

* Electronic address: changan.wang@hzdr.de

$\dagger$ Electronic address: c.h.chang@ifw-dresden.de

[1] Anderson, P. W. Absence of diffusion in certain random lattices. Phys. Rev. 109, 1492-1505 (1958).

[2] Lee, P. A. \& Ramakrishnan, T. V. Disordered electronic systems. Rev. Mod. Phys. 57, 287-337 (1985).

[3] Kramer, B. \& MacKinnon, A. Localization: theory and experiment. Rep. Prog. Phys. 56, 1469 (1993).

[4] Wiersma, D. S., Bartolini, P., Lagendijk, A. \& Righini, R. Localization of light in a disordered medium. Nature 390, 671-673 (1997).

[5] Hu, H., Strybulevych, A., Page, J. H., Skipetrov, S. E. \& van Tiggelen, B. A. Localization of 
ultrasound in a three-dimensional elastic network. Nat. Phys. 4, 945-948 (2008).

[6] Kondov, S. S., McGehee, W. R., Zirbel, J. J. \& DeMarco, B. Three-dimensional anderson localization of ultracold matter. Science 334, 66-68 (2011).

[7] Jendrzejewski, F. et al. Three-dimensional localization of ultracold atoms in an optical disordered potential. Nat. Phys. 8, 398-403 (2012).

[8] Katsumoto, S., Komori, F., Sano, N. \& ichi Kobayashi, S. Fine tuning of metal-insulator transition in al0.3ga0.7as using persistent photoconductivity. J. Phys. Soc. Jpn 56, 22592262 (1987).

[9] Waffenschmidt, S., Pfleiderer, C. \& Löhneysen, H. v. Critical behavior of the conductivity of $\mathrm{Si}: \mathrm{P}$ at the metal-insulator transition under uniaxial stress. Phys. Rev. Lett. 83, 3005-3008 (1999).

[10] Itoh, K. M., Watanabe, M., Ootuka, Y., Haller, E. E. \& Ohtsuki, T. Complete scaling analysis of the metalinsulator transition in ge:ga: Effects of doping-compensation and magnetic field. J. Phys. Soc. Jpn 73, 173-183 (2004).

[11] Möbius, A. The metal-insulator transition in disordered solids: How theoretical prejudices influence its characterization a critical review of analyses of experimental data. Crit. Rev. Solid State Mater. Sci. 0, 1-55 (2018).

[12] Medarde, M. L. Structural, magnetic and electronic properties of $\mathrm{RNiO}_{3}$ perovskites ( $\mathrm{r}=$ rare earth). J. Phys. Condens. Matter 9, 1679 (1997).

[13] Jaramillo, R., Ha, S. D., Silevitch, D. M. \& Ramanathan, S. Origins of bad-metal conductivity and the insulator-metal transition in the rare-earth nickelates. Nat. Phys. 10, 304-307 (2014).

[14] Johnston, S., Mukherjee, A., Elfimov, I., Berciu, M. \& Sawatzky, G. A. Charge disproportionation without charge transfer in the rare-earth-element nickelates as a possible mechanism for the metal-insulator transition. Phys. Rev. Lett. 112, 106404 (2014).

[15] Guo, H. et al. Antiferromagnetic correlations in the metallic strongly correlated transition metal oxide $\mathrm{LaNi}_{3}$. Nat. Commun. 9, 43 (2018).

[16] Scherwitzl, R. et al. Metal-insulator transition in ultrathin $\mathrm{LaNiO}_{3}$ films. Phys. Rev. Lett. 106, 246403 (2011).

[17] Giannazzo, F., Sonde, S., Rimini, E. \& Raineri, V. Lateral homogeneity of the electronic properties in pristine and ion-irradiated graphene probed by scanning capacitance spectroscopy. Nanoscale Res Lett. 6, 109 (2011). 
[18] Guo, H. et al. Strain doping: Reversible single-axis control of a complex oxide lattice via helium implantation. Phys. Rev. Lett. 114, 256801 (2015).

[19] Rowell, J., Dynes, R. \& Schmidt, P. Ion damage, critical current and tunneling studies of $\mathrm{ErRh}_{4} \mathrm{~B}_{4}$ films. In Suhl, H. \& Maple, M. B. (eds.) Superconductivity in D- and F-Band Metals, 409-418 (Academic Press, 1980).

[20] Giuliani, G. F. \& Quinn, J. J. Lifetime of a quasiparticle in a two-dimensional electron gas. Phys. Rev. B 26, 4421-4428 (1982).

[21] Beenakker, C. W. J. \& van Houten, H. Quantum transport in semiconductor nanostructures. Solid State Physics 44, 1-228 (1991).

[22] Gunnarsson, O., Calandra, M. \& Han, J. E. Colloquium: Saturation of electrical resistivity. Rev. Mod. Phys. 75, 1085-1099 (2003).

[23] Hussey, N. E., Takenaka, K. \& Takagi, H. Universality of the mottiofferegel limit in metals. Philos. Mag 84, 2847-2864 (2004).

[24] Wiesmann, H. et al. Simple model for characterizing the electrical resistivity in $a-15$ superconductors. Phys. Rev. Lett. 38, 782-785 (1977).

[25] Gurvitch, M. Ioffe-regel criterion and resistivity of metals. Phys. Rev. B 24, 7404-7407 (1981).

[26] Mikheev, E. et al. Tuning bad metal and non-fermi liquid behavior in a mott material: Rareearth nickelate thin films. Sci. Adv. 1 (2015).

[27] Yoo, H. K. et al. Latent instabilities in metallic $\mathrm{LaNiO}_{3}$ films by strain control of fermi-surface topology. Sci. Rep 5, 8746 (2015).

[28] Subedi, A. Breathing distortions in the metallic, antiferromagnetic phase of $\mathrm{LaNiO}_{3}$. ArXiv e-prints (2017). 1708.08899.

[29] Zhang, J. Y., Kim, H., Mikheev, E., Hauser, A. J. \& Stemmer, S. Key role of lattice symmetry in the metal-insulator transition of $\mathrm{NdNiO}_{3}$ films. Sci. Rep 6, 23652 (2016).

[30] Phanindra, V. E., Agarwal, P. \& Rana, D. S. Terahertz spectroscopic evidence of non-fermiliquid-like behavior in structurally modulated $\mathrm{PrNiO}_{3}$ thin films. Phys. Rev. Materials 2, 015001 (2018).

[31] Baber, W. G. The contribution to the electrical resistance of metals from collisions between electrons. Proc. Royal Soc. A 158, 383-396 (1937).

[32] Rammer, J. \& Schmid, A. Destruction of phase coherence by electron-phonon interactions in disordered conductors. Phys. Rev. B 34, 1352-1355 (1986). 
[33] Wei, H., Jenderka, M., Bonholzer, M., Grundmann, M. \& Lorenz, M. Modeling the conductivity around the dimensionality-controlled metal-insulator transition in $\mathrm{LaNiO}_{3} / \mathrm{LaAlO}_{3}(100)$ superlattices. Appl. Phys. Lett. 106, 042103 (2015).

[34] Herranz, G. et al. Magnetic field effect on quantum corrections to the low-temperature conductivity in metallic perovskite oxides. Phys. Rev. B 72, 014457 (2005).

[35] Kawabata, A. Theory of negative magnetoresistance in three-dimensional systems. Solid State Commun. 34, 431-432 (1980).

[36] Apsley, N. \& Hughes, H. P. Temperature- and field-dependence of hopping conduction in disordered systems, ii. The Philos. Mag: A Journal of Theoretical Experimental and Applied Physics 31, 1327-1339 (1975).

[37] Mott, N. F. Conduction in non-crystalline materials. The Philos. Mag: A Journal of Theoretical Experimental and Applied Physics 19, 835-852 (1969).

[38] Brenig, W., Dhler, G. H. \& Heyszenau, H. Hopping conductivity in highly anisotropic systems. The Philos. Mag: A Journal of Theoretical Experimental and Applied Physics 27, 1093-1103 (1973).

[39] Ortix, C., Kiravittaya, S., Schmidt, O. G. \& van den Brink, J. Curvature-induced geometric potential in strain-driven nanostructures. Phys. Rev. B 84, 045438 (2011).

[40] Chang, C.-H. \& Ortix, C. Ballistic anisotropic magnetoresistance in coreshell nanowires and rolled-up nanotubes. Int. J. Mod. Phys. B 31, 1630016 (2017).

[41] Scherwitzl, R., Zubko, P., Lichtensteiger, C. \& Triscone, J.-M. Electric-field tuning of the metal-insulator transition in ultrathin films of $\mathrm{LaNiO}_{3}$. Appl. Phys. Lett. 95, 222114 (2009).

[42] Pontes, D. S. L. et al. Structural and electrical properties of $\mathrm{LaNi}_{3}$ thin films grown on (100) and (001) oriented srlaalo4 substrates by chemical solution deposition method. MRS Proceedings 1633, 25-33 (2014).

[43] Mott, N. \& Davis, E. Electronic Processes in Non-Crystalline Materials (OUP Oxford, 2012).

[44] Mott, N. The mobility edge since 1967. J. Phys. Condens. Matter 20, 3075 (1987).

[45] Semeghini, G. et al. Measurement of the mobility edge for 3d anderson localization. Nat. Phys. 11, 554-559 (2015).

[46] Pasek, M., Orso, G. \& Delande, D. Anderson localization of ultracold atoms: Where is the mobility edge? Phys. Rev. Lett. 118, 170403 (2017). 